\title{
无过渡金属催化条件下咪唑衍生物与炔基溴的炔基化反应研究
}

\author{
于敬阳彭进松岳亦霞赵宇卓陈春霞* \\ (东北林业大学理学院化学化工系 哈尔滨 150040)
}

\begin{abstract}
摘要 以炔基溴和咪唑衍生物为反应原料, 详细探讨了无过渡金属催化条件下的咪唑类芳香炔胺化合物的合成方法. 研究结果表明, 以 $N, N$-二甲基甲酰胺为溶剂、碳酸钾为碱, 于 $80{ }^{\circ} \mathrm{C}$ 下反应 $12 \mathrm{~h}$ 即可获得中等偏上收率的碳-氮偶联产 物. 通过上述方法合成得到了系列芳香炔胺衍生物, 其结构经 ${ }^{1} \mathrm{H} N \mathrm{NR}$ 和 ${ }^{13} \mathrm{C} N \mathrm{NR}$ 表征.
\end{abstract}

关键词 无过渡金属催化; 咪唑衍生物; 炔基溴; 碳-氮交叉偶联; 芳香炔胺

\section{Transition-Metal-Free Alkynylation of Imidazoles with Alkynyl Bromides}

\author{
Yu, Jingyang Peng, Jinsong Yue, Yixia Zhao, Yuzhuo Chen, Chunxia* \\ (Department of Chemistry and Chemical Engineering, College of Science, Northeast Forestry University, Harbin 150040)
}

\begin{abstract}
Transition-metal-free alkynylation of imidazoles with alkynyl bromides was examined in detail. With $\mathrm{K}_{2} \mathrm{CO}_{3}$ as base in $N, N$-dimethylformamide (DMF) at $80{ }^{\circ} \mathrm{C}$ for $12 \mathrm{~h}$, the reaction afforded the corresponding carbon-nitrogen cross-coupling products in moderate yield. Imidazole-based aromatic ynamines were synthesized by the above method, and the structure was characterized by ${ }^{1} \mathrm{H}$ NMR and ${ }^{13} \mathrm{C}$ NMR spectra.
\end{abstract}

Keywords transition-metal-free; imidazoles; alkynyl bromides; $\mathrm{C}-\mathrm{N}$ cross-coupling; aromatic ynamines

炔胺是一类氮原子与炔基官能团直接相连的重要 化合物，许多芳香炔胺衍生物，如 $N^{9}$-炔基腺嘌呤、7乙炔基茶碱等，具有抗肿瘤 ${ }^{[1]}$ 、抑制磷酸二酯酶活

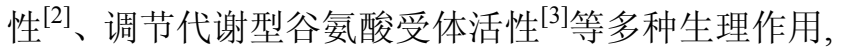
此外, 芳香炔胺还可作为有机中间体应用于材料 ${ }^{[4]}$ 和复 杂分子 ${ }^{[5]}$ 的合成之中, 如图 1 所示. 炔胺化合物的合成 及应用研究一直受到化学家的广泛关注 ${ }^{[6]}$, 常见的炔胺 合成方法包括强碱性条件下杂取代炔化物的亲核取代 反应、胺基化物的消除合成法、炔丙基胺的异构化及炔 胺交换法等 ${ }^{[6,66,6 b]}$. 但是, 这些传统的合成方法普遍存 在收率不高、选择性差和原子经济性低等缺点 ${ }^{[7]}$, 难以 满足学术界和工业界对炔胺类化合物大量制备的要求. 因此, 开发高效、高选择性和原子经济性的温和合成方 法已成为该研究领域的重要内容.

近十年来，过渡金属催化碳一氮交叉偶联反应因条 件温和、反应效率高等特点, 已发展成为一项成熟的催 化技术应用到炔基酰胺化合物的合成中 ${ }^{[8]}$. 近年报道的<smiles>C#Cn1cnc2c(N)ncnc21</smiles>

$N^{9}$-乙炔基腺嘌呤 抗结肠癌生物活性<smiles>C#Cn1cnc2c1c(=O)n(C)c(=O)n2C</smiles>

7-乙炔基茶碱 磷酸二酯酶抑制活性<smiles>[R]C#Cn1c([R])[Z]c2ccccc21</smiles>

$\mathrm{Z}=\mathrm{CH}, \mathrm{N}$

杂环合成筑块<smiles>CC1(C)CCc2nc(C#Cn3cnc(-c4cccnc4)c3)ccc2C1=O</smiles>

炔基咪唑类化合物 代谢型谷氨酸受体调节剂

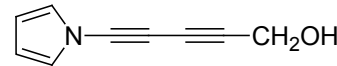

5-吡咯-1-戊-2,4-二炔-1-醇 光电导性材料
图 1 重要芳香炔胺化合物及合成中间体

Figure 1 Important aromatic alkynlamines and synthetic intermediates

铜催化 $\mathrm{N}-\mathrm{H}$ 和端炔化合物的氧化脱氢交叉偶联反应, 可直接利用简单原料一步构建炔胺结构，也有望成为另 一个具有普遍应用性的催化合成方法 ${ }^{[9]}$. 然而，通过铜

*E-mail: ccx1759@163.com

Received January 7, 2014; revised March 25, 2014; published online April 15, 2014.

Project supported by the Scientific Research Fund of Heilongjiang Provincial Education Department (No. 12533023)

黑龙江省教育厅科学技术研究(No. 12533023)资助项目. 
催化方式利用炔基卤代烃的碳-氮偶联反应合成杂芳香 炔胺类化合物时, 目标产物收率普遍较低; 此外, 反应 体系中需要添加 $20 \%$ 左右的具有特殊结构的 $\beta$-二羰基 或邻菲罗啉型有机配体, 既增加了合成成本, 另一方面 也为产物的分离纯化带来了不便 ${ }^{[10]}$. 近年来, 无过渡金 属催化的碱直接促进的碳一碳和碳一杂交叉偶联反应引 起了合成化学家的重视 ${ }^{[11]}$, 我们也一直努力致力于该 领域的研究工作 ${ }^{[12]}$. 在此, 论文拟通过碱促进的咪唑衍 生物与炔基溴直接脱溴化氢来合成芳香炔胺化合物 ${ }^{[13]}$, 如图 2 所示.

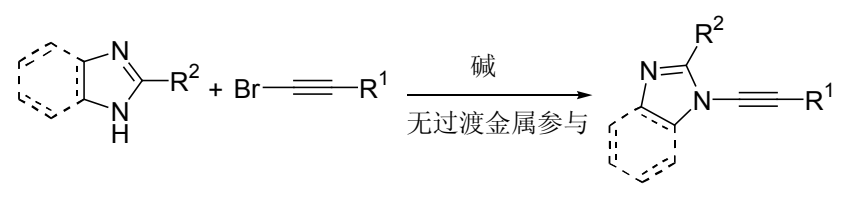

图 2 碱促进的咪唑衍生物与炔基溴的炔基化反应

Figure 2 Base-promoted alkynylation of imidazoles with alkynyl bromides

\section{1 结果与讨论}

\section{1 控制条件篮选与优化}

以 2-苯基苯并咪唑和苯乙炔基溴为底物, 无过渡金 属催化下的反应控制条件优化结果如表 1 所示. 由表 1 可知, 当以碳酸钾为碱, 在 $N, N$-二甲基甲酰胺(DMF)溶 剂中反应 $3 \mathrm{~h}$, 不同温度条件对反应结果影响较大. 在 $80{ }^{\circ} \mathrm{C}$ 条件下反应效果较好, 温度过低, 主反应速率变 慢, 单位时间产率降低; 温度过高, 2-苯基苯并咪唑对 苯乙炔基溴亲核加成反应生成烯基溴副产物 $\mathbf{1 b}$ 会加 强 ${ }^{[14,15]}$, 实验研究也证实 $\mathbf{1 b}$ 的形成并不是通过主产物 $1 \mathrm{a}$ 转化得到的 ${ }^{[10 b, 16]}$, 主产物 1a 收率也会随着温度的上 升而逐渐降低(表 1, Entries 1 6). 为促进该反应的顺利 进行, 溶剂的选择至关重要: 非极性溶剂, 如甲苯和四 氢呋喃(THF), 其它强极性溶剂, 如 $N, N$-二甲基乙酰胺 (DMA)和 $N$-甲基吡咯烷酥(NMP)对反应过程都不利, 无 交叉偶联产物生成(表 1, Entries 7 11). 选择不同碱性 物质如碳酸铯、叔丁醇钾、氢氧化钾和氢氧化钠时, 碱 性较弱的碳酸铯反应效果与碳酸钾相当; 碱性增强, 亲 核加成副反应产率随之上升, 不利于炔基化反应过程 (表 1 , Entries 12 15). 以碳酸钾为碱, $N, N$-二甲基甲酰 胺为溶剂, 在 $80{ }^{\circ} \mathrm{C}$ 条件下研究时间对炔基化过程的影 响表明, 随着反应时间的延长, 产率会随之增加, $12 \mathrm{~h}$ 后产率增加变化不再明显(表 1, Entries 16～19). 关于无 过渡金属参与的偶联反应存在较大争议 ${ }^{[11,17]}$, 近年研究 也表明，偶联反应的发生可能是体系中(如碱、配体或溶 剂)所含痕量过渡金属在起催化作用 ${ }^{[17]}$. 为此, 我们对 $\mathrm{K}_{2} \mathrm{CO}_{3}$ 碱中所含痕量过渡金属进行了检测 ${ }^{[12 a]}$, 其含量
分别为铜 $1 \mathrm{mg} / \mathrm{kg}$, 钯 $2 \mathrm{mg} / \mathrm{kg}$, 镍 $2 \mathrm{mg} / \mathrm{kg}$, 铁 $3 \mathrm{mg} / \mathrm{kg}$. 以 $5 \mathrm{~mol} \% \mathrm{CuI}$ 为催化剂考察过渡金属铜对该化学反应 影响时发现, CuI 的存在提高了亲核加成反应的产率, 碳一氮偶联反应的产率较无 $\mathrm{CuI}$ 时降低了 $15 \%$ (表 1 , Entries 5,20), 此结果也暗示着反应体系中铜的存在对 交叉偶联反应不利. 简言之, 2-苯基苯并咪唑和苯乙炔 基溴在无金属催化条件下进行交叉偶联反应，以 DMF 作溶剂, $\mathrm{K}_{2} \mathrm{CO}_{3}$ 为碱, $80{ }^{\circ} \mathrm{C}$ 下反应 $12 \mathrm{~h}$ 效果较佳.

表 1 苯乙炔基澳与 2-苯基苯并咪唑交叉偶联反应控制条件 筷选与优化 ${ }^{a}$

Table 1 Reaction condition optimization of alkynylation of 2-phenyl-1 $H$-benzo[d]imidazole with (bromoethynyl)benzene

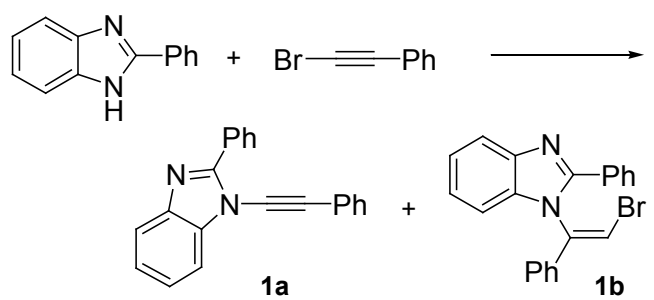

\begin{tabular}{|c|c|c|c|c|c|c|}
\hline \multirow{2}{*}{ Entry } & \multirow{2}{*}{\multicolumn{2}{|c|}{ Temp. $/{ }^{\circ} \mathrm{C}$ Base }} & \multirow{2}{*}{ Solvent } & \multirow{2}{*}{ Time/h } & \multicolumn{2}{|c|}{ Yield $^{b} / \%$} \\
\hline & & & & & $1 \mathrm{a}$ & $1 b$ \\
\hline 1 & 120 & $\mathrm{~K}_{2} \mathrm{CO}_{3}$ & DMF & 3 & 16 & 64 \\
\hline 2 & 110 & $\mathrm{~K}_{2} \mathrm{CO}_{3}$ & DMF & 3 & 27 & 45 \\
\hline 3 & 100 & $\mathrm{~K}_{2} \mathrm{CO}_{3}$ & DMF & 3 & 35 & 32 \\
\hline 4 & 90 & $\mathrm{~K}_{2} \mathrm{CO}_{3}$ & DMF & 3 & 44 & 20 \\
\hline 5 & 80 & $\mathrm{~K}_{2} \mathrm{CO}_{3}$ & DMF & 3 & 47 & 18 \\
\hline 6 & 70 & $\mathrm{~K}_{2} \mathrm{CO}_{3}$ & DMF & 3 & 38 & 14 \\
\hline 7 & 80 & $\mathrm{~K}_{2} \mathrm{CO}_{3}$ & $\mathrm{THF}$ & 3 & 痕量 & 0 \\
\hline 8 & 80 & $\mathrm{~K}_{2} \mathrm{CO}_{3}$ & 甲苯 & 3 & 0 & 0 \\
\hline 9 & 80 & $\mathrm{~K}_{2} \mathrm{CO}_{3}$ & DMA & 3 & 0 & 24 \\
\hline 10 & 80 & $\mathrm{~K}_{2} \mathrm{CO}_{3}$ & 1,4-二氧六环 & 3 & 0 & 22 \\
\hline 11 & 80 & $\mathrm{~K}_{2} \mathrm{CO}_{3}$ & NMP & 3 & 0 & 17 \\
\hline 12 & 80 & $\mathrm{Cs}_{2} \mathrm{CO}_{3}$ & DMF & 3 & 42 & 25 \\
\hline 13 & 80 & $\mathrm{KOtBu}$ & DMF & 3 & 27 & 44 \\
\hline 14 & 80 & $\mathrm{KOH}$ & DMF & 3 & 15 & 39 \\
\hline 15 & 80 & $\mathrm{NaOH}$ & DMF & 3 & 34 & 35 \\
\hline 16 & 80 & $\mathrm{~K}_{2} \mathrm{CO}_{3}$ & DMF & 6 & 50 & 20 \\
\hline 17 & 80 & $\mathrm{~K}_{2} \mathrm{CO}_{3}$ & DMF & 12 & 51 & 22 \\
\hline 18 & 80 & $\mathrm{~K}_{2} \mathrm{CO}_{3}$ & DMF & 24 & 50 & 25 \\
\hline 19 & 80 & $\mathrm{~K}_{2} \mathrm{CO}_{3}$ & DMF & 36 & 49 & 31 \\
\hline $20^{c}$ & 80 & $\mathrm{~K}_{2} \mathrm{CO}_{3}$ & DMF & 3 & 32 & 34 \\
\hline
\end{tabular}

反应条件: 2-苯基苯并咪唑(116 mg, $0.6 \mathrm{mmol})$, 苯乙炔基溴 $(91 \mathrm{mg}, 0.5$ $\mathrm{mmol})$, 碱 $(0.6 \mathrm{mmol})$, 溶剂 $(1.2 \mathrm{~mL}) .{ }^{b}$ 柱色谱分离提纯. ${ }^{c}$ 加入 $5 \mathrm{~mol} \% \mathrm{CuI}$ $(5 \mathrm{mg}, 0.025 \mathrm{mmol})$ 作为催化剂.

\section{2 底物取代基团对化学反应的影响}

\subsection{1 反应适用范围研究}

在获得较佳反应控制条件后，论文探讨了不同取代 的咪唑衍生物与炔基溴底物进行碳一氮交叉偶联反应的 情况, 其研究结果如表 2 所示. 芳香炔基溴与脂肪炔基 溴底物都能有效参与该类化学反应(表 2, Entries 1, 9), 
咪唑或苯并咪唑 2 位上无取代基团时可获得中等以上收 率的芳香炔胺产物(表 2, Entries 1,9); 2 位连有甲基或具 有一定空间位阻的芳基取代基时，产率中等偏下(表 2 , Entries 2 4); 苯并咪唑 2 位为芳基取代基团且在位阻 效应相当的条件下，富电子芳香体系对反应有利(表 2 ,
Entries 5～7); 芳香炔基溴芳香环上连有吸电子取代基 时对反应不利, 吸电子能力越强收率越低(表 2, Entries $1,5,8)$. 此外, 当使用不对称取代苯并咪唑底物(如 5-氯 - $1 H$-苯并咪唑)时, 得到混合产物, 其区域选择性比例接 近 1：1(表 2, Entry 10).

表 2 反应底物对碳-氮交叉偶联反应的影响研究 ${ }^{a}$

Table 2 Effect of imidazoles and alkynyl bromides on the reaction

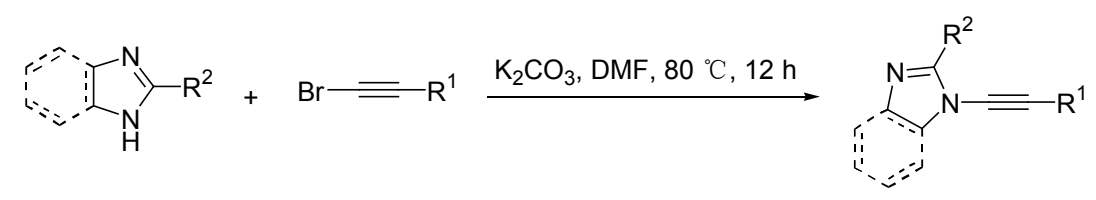

\begin{tabular}{|c|c|c|c|c|}
\hline Entry & Alkynyl bromide & Imidazole & Product & Yield $^{b} / \%$ \\
\hline 1 & $\overline{\overline{B r}}$ & & & $74(65)^{c}$ \\
\hline 2 & & & & 43 \\
\hline 3 & & & & 29 \\
\hline 4 & & & & 27 \\
\hline 5 & & & & 61 \\
\hline 6 & & & & 36 \\
\hline 7 & & & & 71 \\
\hline
\end{tabular}




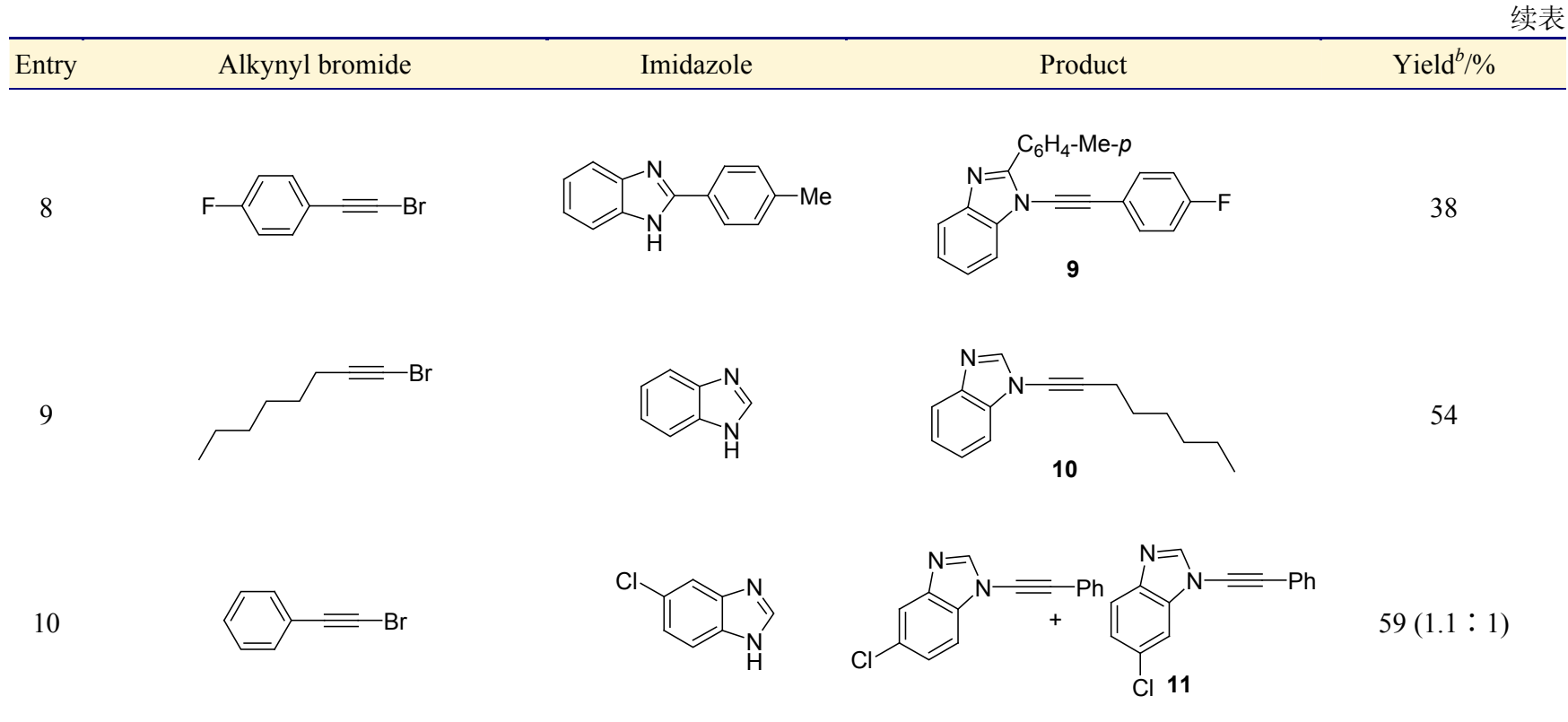

到应条件: 咪唑底物 $0.6 \mathrm{mmol}$, 炔基澳底物 $0.5 \mathrm{mmol}, \mathrm{K}_{2} \mathrm{CO}_{3} 0.6 \mathrm{mmol}, \mathrm{DMF} 1.2 \mathrm{~mL}, 80{ }^{\circ} \mathrm{C}, 12 \mathrm{~h} .^{b}$ 柱色谱分离提纯. ${ }^{c}$ 咪唑 $12.0 \mathrm{mmol}$, 苯乙炔溴 $10.0 \mathrm{mmol}$, $\mathrm{K}_{2} \mathrm{CO}_{3} 12.0 \mathrm{mmol}$, DMF $30 \mathrm{~mL}, 80^{\circ} \mathrm{C}, 72 \mathrm{~h}$.

\section{2 结论}

本文详细研究了咪唑类衍生物与炔基溴在无过渡 金属催化条件下发生的炔基化反应过程，以碳酸钾为 碱, 在 $80{ }^{\circ} \mathrm{C}$ 条件下于 $\mathrm{DMF}$ 溶剂中反应 $12 \mathrm{~h}$ 即可获得中 等产率的咪唑类芳香炔胺类化合物. 在上述标准条件 下，通过改变两种偶联底物的结构合成了系列目标产 物, 探讨了该合成方法的适用范围与局限性. 总之, 在 此报道的合成方法为咪唑类芳香炔胺物质的获得提供 了一条步骤少、成本低、后处理简单和产品收率相对较 高的途径, 为该类化合物的物性和应用研究奠定了基 础.

\section{3 实验部分}

\section{1 仪器与试剂}

${ }^{1} \mathrm{H} \mathrm{NMR}$ 以 $\mathrm{CDCl}_{3}$ 为溶剂, TMS 为内标, 德国 Bruker 公司; MP470 全自动熔点仪, 济南海能仪器公 司. 所有化学试剂均为分析纯, 安耐吉化学, 溶剂经无 水处理.

\section{2 底物及目标产物通用合成方法}

\subsection{1 苯并咪唑类底物的合成}

在装有摚拌子、回流冷凝管的 $50 \mathrm{~mL}$ 圆底烧瓶中, 依次加入邻苯二胺 $(0.277 \mathrm{~g}, 2.5 \mathrm{mmol})$ 、苯甲酸 $(0.61 \mathrm{~g}, 5$ $\mathrm{mmol}$ )和 $20 \mathrm{~mL}$ 乙二醇, 加热到 $190{ }^{\circ} \mathrm{C}$ 下回流 $3 \mathrm{~h}$, 待反 应混合物冷却至室温后加入 $25 \mathrm{~mL}$ 蒸馏水, 有黄色固体 析出, 抽滤、洗涤, 干燥, $95 \%$ 乙醇重结晶, 得到目标产 物 2-苯基苯并咪唑 ${ }^{[18]} 0.42 \mathrm{~g}$, 收率 $86 \%$. m.p. 295 297
${ }^{\circ} \mathrm{C} ;{ }^{1} \mathrm{H}$ NMR $\left(\mathrm{CDCl}_{3}, 400 \mathrm{MHz}\right) \delta: 12.92(\mathrm{~s}, 1 \mathrm{H}, \mathrm{NH})$, $8.20 \sim 8.18(\mathrm{~m}, 2 \mathrm{H}), 7.62 \sim 7.57(\mathrm{~m}, 2 \mathrm{H}), 7.53 \sim 7.43(\mathrm{~m}$, $3 \mathrm{H}), 7.21 \sim 7.18(\mathrm{~m}, 2 \mathrm{H}) ;{ }^{13} \mathrm{C} \mathrm{NMR}\left(\mathrm{CDCl}_{3}, 100 \mathrm{MHz}\right) \delta$ : $150.8,137.9,137.9,131.8,129.4,127.8,125.7,123.1$, $122.8,121.3,121.1,116.8,111.2$.

\subsection{2 炔基溴底物的合成}

在 $50 \mathrm{~mL}$ 圆底烧瓶中依次加入苯乙炔(1.532 g, 15 mmol), NBS (2.94 g, $16.5 \mathrm{mmol}), \mathrm{AgNO}_{3}(0.25 \mathrm{~g}, 1.5$ $\mathrm{mmol}$ )和 $30 \mathrm{~mL}$ 丙酮, 室温下搅拌反应 $3 \mathrm{~h}$, 浓缩, 柱色 谱分离提纯(洗脱剂: 石油醚)得无色液体 ${ }^{[16]} 2.67 \mathrm{~g}$, 产 率 98.5\%. ${ }^{1} \mathrm{H}$ NMR $\left(\mathrm{CDCl}_{3}, 400 \mathrm{MHz}\right) \delta: 7.45 \sim 7.42(\mathrm{~m}$, $2 \mathrm{H}), 7.25(\mathrm{~s}, 1 \mathrm{H}), 7.24 \sim 7.22(\mathrm{~m}, 2 \mathrm{H}) ;{ }^{13} \mathrm{C} \mathrm{NMR}\left(\mathrm{CDCl}_{3}\right.$, $100 \mathrm{MHz}) \delta$ : 128.5, 128.4, 128.4, 132.4, 132.4, 122.8, 77.4, 75.3 .

\subsection{3 芳香炔胺产物的合成}

在 $10 \mathrm{~mL}$ 密封反应管中依次加入 2-苯基苯并咪唑 $(116 \mathrm{mg}, 0.6 \mathrm{mmol}) 、$ 苯乙炔基澳 $(91 \mathrm{mg}, 0.5 \mathrm{mmol}) 、$ 碳 酸钾 $(83 \mathrm{mg}, 0.6 \mathrm{mmol}$ )和 $1.2 \mathrm{~mL}$ 无水 $\mathrm{DMF}$ 溶剂, 于 80 ${ }^{\circ} \mathrm{C}$ 下反应 $6 \mathrm{~h}$. 待反应混合物冷却至室温后, 加入 $5 \mathrm{~mL}$ 蒸馏水, 乙酸乙酯萃取三次合并, 饱和食盐水洗涤, 无 水硫酸镁干燥，过滤，浓缩，柱层析分离[硅胶，洗脱剂 为 $V$ (石油醚) $: V$ (乙酸乙酯 $)=20: 1$ 的混合溶剂]得白色 固体 $74 \mathrm{mg}$, 产率 $51 \%$. m.p. $193 \sim 195{ }^{\circ} \mathrm{C} ;{ }^{1} \mathrm{H}$ NMR $\left(\mathrm{CDCl}_{3}, 400 \mathrm{MHz}\right) \delta: 7.81 \sim 7.79(\mathrm{~m}, 2 \mathrm{H}), 7.49 \sim 7.48(\mathrm{~m}$, $1 \mathrm{H}), 7.43 \sim 7.42(\mathrm{~m}, 1 \mathrm{H}), 7.33 \sim 7.32(\mathrm{~m}, 1 \mathrm{H}), 7.32 \sim 7.31$ (m, 1H), $7.25 \sim 7.24(\mathrm{~m}, 2 \mathrm{H}), 7.26 \sim 7.25(\mathrm{~m}, 2 \mathrm{H}), 7.24$ $(\mathrm{m}, 1 \mathrm{H}), 7.23 \sim 7.22(\mathrm{~m}, 1 \mathrm{H}), 7.22 \sim 7.21(\mathrm{~m}, 2 \mathrm{H}) ;{ }^{13} \mathrm{C}$ 
NMR $\left(\mathrm{CDCl}_{3}, 100 \mathrm{MHz}\right) \delta: 152.1,141.6,135.9,131.5$, $131.2,129.2,128.9,128.6,128.5,128.2,124.7,124.6$, 120.7, 120.0, 111.2, 77.4, 75.3.

\section{3 产物表征分析}

(Z)-1-(1-苯基-2-溴乙烯基)-2-苯基苯并咪唑(1b $)^{[14]}$ : 白色固体, m.p. $151 \sim 153{ }^{\circ} \mathrm{C} ;{ }^{1} \mathrm{H} \mathrm{NMR}\left(\mathrm{CDCl}_{3}, 400 \mathrm{MHz}\right)$ $\delta: 7.94(\mathrm{~d}, J=8.0 \mathrm{~Hz}, 1 \mathrm{H}), 7.84 \sim 7.81(\mathrm{~m}, 2 \mathrm{H}), 7.41 \sim$ $7.37(\mathrm{~m}, 4 \mathrm{H}), 7.36 \sim 7.31(\mathrm{~m}, 4 \mathrm{H}), 7.29(\mathrm{~s}, 1 \mathrm{H}), 7.24 \sim$ $7.15(\mathrm{~m}, 3 \mathrm{H}) ;{ }^{13} \mathrm{C}$ NMR $\left(\mathrm{CDCl}_{3}, 100 \mathrm{MHz}\right) \delta: 142.3$, $141.5,138.7,131.2,130.9,130.8,129.6,128.9,128.8$, $128.2,127.7,126.7,123.3,123.1,115.5,115.3,80.7$. HRMS-ESI calcd for $\mathrm{C}_{21} \mathrm{H}_{15} \mathrm{BrN}_{2} \mathrm{Na}[\mathrm{M}+\mathrm{Na}]^{+} 397.0316$; found 397.0319 .

1-(2-苯基乙炔基)咪唑(2) ${ }^{[10 b]}$ : 白色固体, m.p. 42 $43{ }^{\circ} \mathrm{C} ;{ }^{1} \mathrm{H}$ NMR $\left(\mathrm{CDCl}_{3}, 400 \mathrm{MHz}\right) \delta: 7.84(\mathrm{~s}, 1 \mathrm{H}), 7.54 \sim$ $7.50(\mathrm{~m}, 2 \mathrm{H}), 7.39 \sim 7.36(\mathrm{~m}, 3 \mathrm{H}), 7.22(\mathrm{~s}, 1 \mathrm{H}), 7.11(\mathrm{~s}$, $1 \mathrm{H}) ;{ }^{13} \mathrm{C}$ NMR $\left(\mathrm{CDCl}_{3}, 100 \mathrm{MHz}\right) \delta: 140.0,131.7,129.3$, 129.0, 128.5, 121.7, 121.0, 78.1, 70.3.

2-甲基-1-(2-苯基乙炔基)咪唑(3) ${ }^{[10 b]}$ : 淡黄色固体, m.p. $32 \sim 33{ }^{\circ} \mathrm{C} ;{ }^{1} \mathrm{H}$ NMR $\left(\mathrm{CDCl}_{3}, 400 \mathrm{MHz}\right) \delta$ : $7.49 \sim$ $7.44(\mathrm{~m}, 2 \mathrm{H}), 7.35 \sim 7.31(\mathrm{~m}, 3 \mathrm{H}), 7.05(\mathrm{~d}, J=1.6 \mathrm{~Hz}$, $1 \mathrm{H}), 6.88(\mathrm{~d}, J=1.6 \mathrm{~Hz}, 1 \mathrm{H}), 2.51(\mathrm{~s}, 3 \mathrm{H}) ;{ }^{13} \mathrm{C} \mathrm{NMR}$ $\left(\mathrm{CDCl}_{3}, 100 \mathrm{MHz}\right) \delta$ : 148.6, 131.4, 128.6, 128.3, 127.6, 121.2, 121.1, 78.1, 72.5, 13.2.

2-(2-甲氧基苯基)-1-(2-苯基乙炔基)苯并咪唑(4): 棕色固体, m.p. $76 \sim 78{ }^{\circ} \mathrm{C} ;{ }^{1} \mathrm{H} \mathrm{NMR}\left(\mathrm{CDCl}_{3}, 400 \mathrm{MHz}\right) \delta$ : $8.30 \sim 8.26(\mathrm{~m}, 2 \mathrm{H}), 7.82 \sim 7.80(\mathrm{~m}, 1 \mathrm{H}), 7.66 \sim 7.63(\mathrm{~m}$, $1 \mathrm{H}), 7.57 \sim 7.51(\mathrm{~m}, 2 \mathrm{H}), 7.41 \sim 7.35(\mathrm{~m}, 5 \mathrm{H}), 7.07 \sim 7.04$ $(\mathrm{m}, 2 \mathrm{H}), 3.89$ (s, 3H); ${ }^{13} \mathrm{C} \mathrm{NMR}\left(\mathrm{CDCl}_{3}, 100 \mathrm{MHz}\right) \delta$ : $161.6,152.9,142.2,136.6,132.3,131.5,130.9,130.3$, 128.7, 128.6, 124.3, 124.0, 121.8, 121.5, 119.9, 114.1, $110.9,78.1,75.4,55.4$. HRMS-ESI calcd for $\mathrm{C}_{22} \mathrm{H}_{16} \mathrm{~N}_{2} \mathrm{Na}$ $\mathrm{O}[\mathrm{M}+\mathrm{Na}]^{+} 347.1160$; found 347.1162 .

2-苯基-1-[2-(2-氯苯基)乙炔基]咪唑(5): 白色固体, m.p. $67 \sim 69{ }^{\circ} \mathrm{C} ;{ }^{1} \mathrm{H}$ NMR $\left(\mathrm{CDCl}_{3}, 400 \mathrm{MHz}\right) \delta: 8.17 \sim$ $8.14(\mathrm{~m}, 2 \mathrm{H}), 7.49 \sim 7.42(\mathrm{~m}, 5 \mathrm{H}), 7.32 \sim 7.23(\mathrm{~m}, 3 \mathrm{H})$, $7.17 \sim 7.16(\mathrm{~m}, 1 \mathrm{H}) ;{ }^{13} \mathrm{C}$ NMR $\left(\mathrm{CDCl}_{3}, 100 \mathrm{MHz}\right) \delta$ : $149.5,135.8,133.1,129.8,129.6,129.5,129.3,128.9$, $128.8,128.5,128.0,126.6,123.9,121.6,83.9,69.5$. HRMS-ESI calcd for $\mathrm{C}_{17} \mathrm{H}_{11} \mathrm{ClN}_{2} \mathrm{Na}[\mathrm{M}+\mathrm{Na}]^{+} 301.0508$; found 301.0511.

2-苯基-1-[2-(2-氯苯基)乙炔基]苯并咪唑(6): 白色 固体, m.p. $87 \sim 89{ }^{\circ} \mathrm{C} ;{ }^{1} \mathrm{H}$ NMR $\left(\mathrm{CDCl}_{3}, 400 \mathrm{MHz}\right) \delta$ : $8.35 \sim 8.33(\mathrm{~m}, 2 \mathrm{H}), 7.86 \sim 7.84(\mathrm{~m}, 1 \mathrm{H}), 7.75 \sim 7.73(\mathrm{~m}$, $1 \mathrm{H}), 7.55 \sim 7.51(\mathrm{~m}, 4 \mathrm{H}), 7.47 \sim 7.38(\mathrm{~m}, 3 \mathrm{H}), 7.32 \sim 7.24$ $(\mathrm{m}, 2 \mathrm{H}) ;{ }^{13} \mathrm{C}$ NMR $\left(\mathrm{CDCl}_{3}, 100 \mathrm{MHz}\right) \delta: 152.7,142.2$, $136.6,135.4,132.8,130.8,129.6,129.5,128.9,128.8$, $128.6,126.7,124.6,121.9,120.4,111.3,82.5,72.9$. HRMS-ESI calcd for $\mathrm{C}_{21} \mathrm{H}_{13} \mathrm{ClN}_{2} \mathrm{Na}[\mathrm{M}+\mathrm{Na}]^{+} 351.0665$; found 351.0669 .

2-(4-氯苯基)-1-[2-(2-氯苯基)乙炔基]苯并咪唑(7): 黄色固体, m.p. $92 \sim 94{ }^{\circ} \mathrm{C} ;{ }^{1} \mathrm{H} \mathrm{NMR}\left(\mathrm{CDCl}_{3}, 400 \mathrm{MHz}\right) \delta$ : $8.33 \sim 8.31(\mathrm{~d}, J=8.8 \mathrm{~Hz}, 2 \mathrm{H}), 7.85 \sim 7.82(\mathrm{~m}, 1 \mathrm{H})$, $7.74 \sim 7.72(\mathrm{~m}, 1 \mathrm{H}), 7.55 \sim 7.51(\mathrm{~m}, 3 \mathrm{H}), 7.49 \sim 7.47(\mathrm{~m}$, $1 \mathrm{H}), 7.44 \sim 7.38(\mathrm{~m}, 2 \mathrm{H}), 7.35 \sim 7.29(\mathrm{~m}, 2 \mathrm{H}) ;{ }^{13} \mathrm{C} \mathrm{NMR}$ $\left(\mathrm{CDCl}_{3}, 100 \mathrm{MHz}\right) \delta: 151.6,142.1,137.0,136.6,135.5$, $132.8,130.1,129.8,129.6,128.9,127.3,126.7,124.8$, 124.7, 121.7, 120.4, 111.3, 82.2, 73.2. HRMS-ESI calcd for $\mathrm{C}_{21} \mathrm{H}_{12} \mathrm{Cl}_{2} \mathrm{~N}_{2} \mathrm{Na}[\mathrm{M}+\mathrm{Na}]^{+}$385.0275; found 385.0278.

2-(4-甲氧苯基)-1-[2-(2-氯苯基)乙炔基]苯并咪唑 (8): 白色固体, m.p. 97 99 ${ }^{\circ} \mathrm{C} ;{ }^{1} \mathrm{H}$ NMR $\left(\mathrm{CDCl}_{3}, 400\right.$ MHz) $\delta: 8.34 \sim 8.32(\mathrm{~d}, J=8.8 \mathrm{~Hz}, 2 \mathrm{H}), 7.82 \sim 7.80(\mathrm{~m}$, $1 \mathrm{H}), 7.72 \sim 7.70(\mathrm{~m}, 1 \mathrm{H}), 7.56 \sim 7.54(\mathrm{~m}, 1 \mathrm{H}), 7.49 \sim 4.47$ (m, 1H), $7.42 \sim 7.36(\mathrm{~m}, 2 \mathrm{H}), 7.34 \sim 7.28(\mathrm{~m}, 2 \mathrm{H}), 7.07 \sim$ $7.04(\mathrm{~d}, J=9.2 \mathrm{~Hz}, 2 \mathrm{H}), 3.90(\mathrm{~s}, 3 \mathrm{H}) ;{ }^{13} \mathrm{C}$ NMR $\left(\mathrm{CDCl}_{3}\right.$, $100 \mathrm{MHz}) \delta: 166.7,142.5,136.5,132.7,132.5,131.6$, $131.4,129.5,129.2,129.0,128.7,128.3,125.1,124.8$, 121.3, 120.6, 111.6, 77.8, 75.9, 56.0. HRMS-ESI calcd for $\mathrm{C}_{22} \mathrm{H}_{15} \mathrm{ClN}_{2} \mathrm{NaO} \quad[\mathrm{M}+\mathrm{Na}]^{+}$381.0771; found 381.0773 .

2-(4-甲基苯基)-1-[2-(4-氟苯基)乙炔基]苯并咪唑 (9): 棕色固体, m.p. $84 \sim 86{ }^{\circ} \mathrm{C} ;{ }^{1} \mathrm{H}$ NMR $\left(\mathrm{CDCl}_{3}, 400\right.$ MHz) $\delta: 8.19 \sim 8.17(\mathrm{~d}, J=8.4 \mathrm{~Hz}, 2 \mathrm{H}), 7.84 \sim 7.81(\mathrm{~m}$, $1 \mathrm{H}), 7.73 \sim 7.71(\mathrm{~m}, 1 \mathrm{H}), 7.65 \sim 7.63(\mathrm{~m}, 2 \mathrm{H}), 7.55 \sim 7.51$ (m, 2H), $7.42 \sim 7.34(\mathrm{~m}, 2 \mathrm{H}), 7.12 \sim 7.07(\mathrm{~m}, 2 \mathrm{H}), 2.45(\mathrm{~s}$, $3 \mathrm{H}) ;{ }^{13} \mathrm{C}$ NMR $\left(\mathrm{CDCl}_{3}, 100 \mathrm{MHz}\right) \delta: 163.7,153.1,142.2$, $141.1,136.6,133.5,129.4,128.6,126.1,124.4,124.2$, $120.2,117.8,116.0,115.9,111.0,77.7,74.4,21.6$. HRMS-ESI calcd for $\mathrm{C}_{22} \mathrm{H}_{15} \mathrm{FN}_{2} \mathrm{Na}[\mathrm{M}+\mathrm{Na}]^{+} 349.1117$; found 349.1121 .

1-(辛-1-炔基)-苯并咪唑(10) ${ }^{[10 \mathrm{a}]}$ : 黄色粘稠液体. ${ }^{1} \mathrm{H}$ $\operatorname{NMR}\left(\mathrm{CDCl}_{3}, 400 \mathrm{MHz}\right) \delta$ : 8.02 (s, 1H), 7.78 (d, $J=7.6$ $\mathrm{Hz}, 1 \mathrm{H}), 7.53(\mathrm{~d}, J=7.5 \mathrm{~Hz}, 1 \mathrm{H}), 7.36(\mathrm{td}, J=7.5,1.1 \mathrm{~Hz}$, $1 \mathrm{H}), 7.32(\mathrm{td}, J=7.6,1.3 \mathrm{~Hz}, 1 \mathrm{H}), 2.46(\mathrm{t}, J=7.0 \mathrm{~Hz}, 2 \mathrm{H})$, $1.64(\mathrm{q}, J=7.2 \mathrm{~Hz}, 2 \mathrm{H}), 1.52 \sim 1.42(\mathrm{~m}, 2 \mathrm{H}), 1.38 \sim 1.26$ (m, 4H), 0.90 (t, $J=6.9 \mathrm{~Hz}, 3 \mathrm{H}) ;{ }^{13} \mathrm{C}$ NMR $\left(\mathrm{CDCl}_{3}, 100\right.$ MHz) $\delta: 143.9,141.8,134.7,124.4,123.6,120.5,110.8$, 73.6, 68.0, 31.2, 28.5, 28.5, 22.4, 18.3, 14.0.

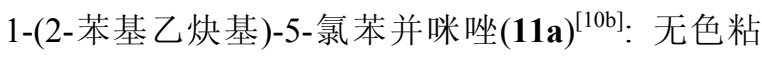
稠液体. ${ }^{1} \mathrm{H}$ NMR $\left(\mathrm{CDCl}_{3}, 400 \mathrm{MHz}\right) \delta: 8.18(\mathrm{~s}, 1 \mathrm{H}), 7.85$ 
$(\mathrm{d}, J=1.6 \mathrm{~Hz}, 1 \mathrm{H}), 7.63 \sim 7.60(\mathrm{~m}, 3 \mathrm{H}), 7.46 \sim 7.42(\mathrm{~m}$, $4 \mathrm{H}) ;{ }^{13} \mathrm{C} \mathrm{NMR}\left(\mathrm{CDCl}_{3}, 100 \mathrm{MHz}\right) \delta: 144.7,142.8,133.2$, $131.8,129.8,129.2,128.6,125.4,121.1,120.8,111.7$, $75.9,73.8$.

1-(2-苯基乙炔基)-6-氯苯并咪唑(11b $)^{[10 b]}$ : 无色粘 稠液体. ${ }^{1} \mathrm{H} \mathrm{NMR}\left(\mathrm{CDCl}_{3}, 400 \mathrm{MHz}\right) \delta: 8.18(\mathrm{~s}, 1 \mathrm{H}), 7.77$ $(\mathrm{d}, J=8.4 \mathrm{~Hz}, 1 \mathrm{H}), 7.70(\mathrm{~d}, J=1.8 \mathrm{~Hz}, 1 \mathrm{H}), 7.63 \sim 7.60$ $(\mathrm{m}, 2 \mathrm{H}), 7.46 \sim 7.43(\mathrm{~m}, 3 \mathrm{H}), 7.37(\mathrm{dd}, J=8.4,1.8 \mathrm{~Hz}$, $1 \mathrm{H}) ;{ }^{13} \mathrm{C} \mathrm{NMR}\left(\mathrm{CDCl}_{3}, 100 \mathrm{MHz}\right) \delta: 144.2,140.4,133.2$, $131.9,130.8,129.3,128.6,125.0,121.7,120.8,111.2$, $75.6,74.1$.

\section{References and note}

[1] (a) Joshi, R. V.; Xu, Z.-Q.; Ksebati, M. B.; Kessel, D.; Corbertt, T. H.; Drach, J. C.; Zemlicka, J. J. Chem. Soc., Perkin Trans. 1 1994, 1089.

(b) Phadtare, S.; Kessel, D.; Corbett, T. H.; Renis, H. E.; Court, B. A.; Zemlicka, J. J. Med. Chem. 1991, 34, 421.

[2] Reisch, J.; Seeger, U. Arch. Pharm. 1977, 310, 851.

[3] (a) Parsons, C. G. R.; Jirgensons, A.; Jaunzeme, I.; Kalvinsh, I.; Henrich, M.; Vanejevs, M.; Weil, T.; Kauss, V.; Danysz, W.; Jatzke, C. WO 2007023290, 2007 [Chem. Abstr. 2007, 147, 118074].

(b) Himmelsback, F.; Hauel, N.; Langkopf, E.; Eckhard, M.; Kauffmann-Hefer, I.; Tadayyon, M.; Thomas, L. WO 2005058901, 2005 [Chem. Abstr. 2005, 145, 88317].

[4] Paley, M. S.; Frazier, D. O.; Abeledeyem, H.; McManus, S. P.; Zutaut, S. E. J. Am. Chem. Soc. 1992, 114, 3247.

[5] (a) Townsend, S. D.; Sulikowski, G. A. Org. Lett. 2013, 15, 5096. (b) Chen, Z.; Zeng, W.; Jiang, H.; Liu, L. Org. Lett. 2012, 14, 5385. (c) Yamasaki, R.; Terashima, N.; Sotome, I.; Komagawa, S.; Saito, S. J. Org. Chem. 2010, 75, 480.

(d) Xu, H.; Zhang, Y.; Huang, J.; Chen, W. Org. Lett. 2010, 12, 3704.

(d) Laroche, C.; Kerwin, S. M. J. Org. Chem. 2009, 74, 9229.

(e) Nadipuram, A. K.; Kerwin, S. M. Tetrahedron 2006, 62, 3798.

[6] (a) Ficini, J. Tetrahedron 1976, 32, 1449.

(b) Zificsak, C. A.; Mulder, J. A.; Hsung, R. P.; Rameshkumar, C.; Wei, L.-L. Tetrahedron 2001, 57, 7575.

(c) Evano, G.; Coste, A.; Jouvin, K. Angew. Chem., Int. Ed. 2010, 49, 2840.

(d) DeKorver, K. A.; Li, H.; Lohse, A. G.; Hayashi, R.; Lu, Z.; Zhang, Y.; Hsung, R. P. Chem. Rev. 2010, 110, 5064.

(e) Wang, X.-N.; Yeom, H.-S.; Fang, L.-C.; He, S.-Z.; Ma, Z.-X.; Kedrowski, B. L.; Hsung, R. P. Acc. Chem. Res. 2014, 47, 560.

[7] He, C.-X. Chem. Reag. 1992, 14, 291 (in Chinese).

(贺灿欣, 化学试剂, 1992, 14, 291.)
[8] (a) Evano, G.; Jouvin, K.; Coste, A. Synthesis 2013, 45, 17.

(b) Priebbenow, D. L.; Becker, P.; Bolm, C. Org. Lett. 2013, 15, 6155.

(c) DeKorver, K. A.; Walton, M. C.; North, T. D.; Hsung, R. P. Org. Lett. 2011, 13, 4862.

(d) Sueda, T.; Oshima, A.; Teno, N. Org. Lett. 2011, 13, 3996.

(e) Jouvin, K.; Couty, F.; Evano, G. Org. Lett. 2010, 12, 3272.

(f) Jia, W.; Jiao, N. Org. Lett. 2010, 12, 2000.

(g) Yao, B.; Liang, Z.; Niu, T.; Zhang, Y. J. Org. Chem. 2009, 74, 4630.

(h) Dooleweerdt, K.; Birkedal, H.; Ruhland, T.; Skrydstrup, T. $J$. Org. Chem. 2008, 73, 9447.

(i) Frederick, M. O.; Mulder, J. A.; Tracey, M. R.; Hsung, R. P.; Huang, J.; Kurtz, K. C. M.; Shen, L.; Douglas, C. J. J. Am. Chem. Soc. 2003, 125, 2368.

[9] (a) Wang, L.; Huang, H.; Priebbenow, D. L.; Pan, F. F.; Bolm, C. Angew. Chem., Int. Ed. 2013, 52, 3478.

(b) Laouiti, A.; Rammah, M. M.; Rammah, M. B.; Marrot, J.; Couty, F.; Evano, G. Org. Lett. 2012, 14, 6.

(c) Hamada, T.; Ye, X.; Stahl, S. S. J. Am. Chem. Soc. 2008, 130, 833.

[10] (a) Laroche, C.; Li, J.; Freyer, M. W.; Ketwin, S. M. J. Org. Chem. 2008, 73, 6462.

(b) Burley, G. A.; Davies, D. L.; Griffith, G. A.; Lee, M.; Sing, K. J. Org. Chem. 2010, 75, 980.

(c) Das, B.; Salvanna, N.; Reddy, G. C.; Balasubramanyam, P. Tetrahedron Lett. 2011, 52, 6497.

(d) Zhang, Y.; Hsung, R. P.; Tracey, M. R.; Kurtz, K. C. M.; Vera, E. L. Org. Lett. 2004, 6, 1151.

[11] (a) Mehta, V. P.; Punji, B. RSC Adv. 2013, 3, 11957.

(b) Arancon, R. A. D.; Lin, C. S. K.; Vargas, C.; Luque, R. Org. Biomol. Chem. 2014, 12, 10.

[12] (a) Chen, C.; Chen, C.; Li, B.; Tao, J.; Peng, J. Molecules 2012, 17 , 12506.

(b) Peng, J.; Zong, C.; Ye, M.; Chen, T.; Gao, D.; Wang, Y. Org. Biomol. Chem. 2011, 9, 1225.

[13] Wu, Z.; Pan, Y.; Zhou, X. Synthesis 2011, 2255.

[14] Peng, J.; Shang, G.; Chen, C.; Miao, Z.; Li, B. J. Org. Chem. 2013, $78,1242$.

[15] Yamagishi, M.; Okazaki, J.; Nishigai, K.; Hata, T.; Urabe, H. Org. Lett. 2012, 14, 34.

[16] Under our standard conditons, a bromide source $(\mathrm{NaBr}, \mathrm{KBr}$ or $\mathrm{Bu}_{4} \mathrm{NBr}$ ) can't react with $\mathbf{1 a}$ even through prolonging reaction time and increasing reaction temperature. 1a was completely recovered.

[17] (a) Buchwald, S. L.; Bolm, C. Angew. Chem., Int. Ed. 2009, 48, 5586 .

(b) Thomé, I.; Nijs, A.; Bolm, C. Chem. Soc. Rev. 2012, 41, 979.

(c) Leadbeater, N. E. Nat. Chem. 2010, 2, 1007.

[18] Li, Y.; Ma, H.-Q.; Wang, Y.-L. Chin. J. Org. Chem. 2008, 28, 210 (in Chinese).

(李炎, 马会强, 王玉炉, 有机化学, 2008, 28, 210.) 